\title{
How Ethical Debates Can Enrich Data Science and Artificial Intelligence
}

\begin{abstract}
Anna Laesser*
The Startup Digest section introduces startups and grassroots initiatives from around the world that push the boundaries of emerging technologies. Most conversations around emerging technologies are stuck in silos and are quite hyped, making it hard to understand their actual impact on businesses, society and governance. The Startup Digests aim to demystify what is happening on the ground by establishing a discourse via case studies and interviews with startups and grassroots initiatives. Each edition will take a critical look on how these movements apply emerging technologies to achieve a specific purpose - facilitating a discourse that makes the (new) thinking, the approach and potential impact become more tangible.
\end{abstract}

\section{Introduction}

Emerging technology will profoundly change how we live tomorrow. Data-based technological solutions have increasingly become part of our cultural identity, transforming the way we communicate, learn, do banking - even date. Although it is revolutionising how we life and work, we have also encountered algorithms that turned racist, misogynistic and homophobic; thereby negatively influencing society on an ethical and political level. Building impactful solutions is not just a matter of data science but one that requires greater care.

This Startup Digest takes a closer look at how two female-led startups in London and Berlin are working with data science and artificial intelligence (AI) to build solutions that maximise a positive impact and minimise harm to society. Both case studies will reveal how they deal with potential benefits and risks and how they take ethical considerations into account.

\section{Interview 1: Dataconomy - Data Science and the Art of Making Data Useful}

Name of your startup/ initiative: Dataconomy Media \& Data Natives

DOI: 10.21552/delphi/2019/2/10

* Anna Laesser, Co-founder at Impact Hub Berlin. All views are my own. For correspondence: <laesser@lexxion.eu>
Location: Berlin

Your name, position: Elena Poughia, Managing Director

Question: How do you create a global community of data driven pioneers and what role does Dataconomy play? Why is it important and what is the vision you are aiming for?

'Create' is not the right word here - You don't really 'create' a global community, you facilitate, you nurture it, you belong, you are part of it. Data Natives (the community arm of Dataconomy) spot a 'movement', a network effect if you will, a moment of collective unconsciousness as coined by Carl Jung. There is a need for people in Europe to learn more about data science, data driven technology and to work on projects together. Until now, $62 \%$ of our community is interested in coming to our events in order to find people to co-create and $80 \%$ are interested in learning new things. Our role is to facilitate that environment, to provide the right tools and to curate the content and the people so we can ensure that all attendees are satisfied. We aspire to be the number one community for the data driven generation; an environment for creators and doers to work on things together. This is very important in order to foster innovation, creation and to encourage the doers to keep on doing.

Question: Can you outline how you/Dataconomy defines data science and why it has become so impor- 
tant? Building on this, what is the difference between digital and data experts?

Data science is the art of making data useful, and it is a difficult and tedious task - currently on the internet every 60 seconds 100 videos are uploaded on YouTube, $40 \mathrm{~K}$ pictures are being posted on instagram, $350 \mathrm{~K}$ tweets are being shared; data science makes it actionable, provides a story and puts this in a framework. Data has always been important - indeed, information is an important evolutionary advancement of civilisation. Ancient Greeks' stories, myths, wisdom and learnings were passed onto rocks and papyrus, the earliest known paper document in Europe was found in the 11th century and by the $15^{\text {th }}$ century, documentation and knowledge passing through books brought about a renaissance in the sciences and philosophy. Computers and information technology are the 'game-changers' of our times. They are transforming the way we communicate, interact, solve problems, work and live our lives.

From a philosophical standpoint, if you think about it, we are all made out of data - what computers have is the information as the algorithmic DNA of their computational power, our DNA contains information we're not even aware of and can not even decode verbally or consciously. It provides us with the unspoken wisdom and the 'gut feeling' of situations to engage or not engage with.

Digital and data experts - that's a very broad question and assumption. Not all data experts are the same as well as not all digital experts do the same thing - when it comes to developers and data scientists, simply put, you need data as a developer but that is not a crucial part of the job, and you need some coding skills as a data scientist however unless you are an engineer you don't need coding as crucial part of the job.

Question: 'Data is neutral' - what is your response? According to you what are the three key most crucial aspects that should be taken into consideration when designing new, ethical data-based solutions?

'Data is neutral' is as broad and generic of a term such as 'tech is good' or 'facts matter'. It is all relative and it all depends on what kind of data, how has it been collected, where there any parameters set and how is it being interpreted. Data is not neutral as long people are not neutral and we all have our own set of bi- ases that have been culturally and socially constructed. How we interpret the world depends on who we are - gender, sex, age, nationality, race, class and all other cultural and social factors.

When designing new systems one should take into consideration the data being used and include an explanation to justify any given biases. Many advocate for 'explainable' AIs - systems that trace back to the 'how' a system decided on a topic. Based on what we would like to achieve, it would be good to have diverse data samples and ensure that we have a diverse team of people assessing the results.

Question: Data is part of our new cultural identity, transforming the way we communicate, learn, do banking, even date. Data-based solutions have massive potential to change our lives. However, there is a great responsibility to design ethical solutions that maximise positive impact and minimise harm to society. Does Dataconomy address ethical debates? If yes, how do you empower people to design positive solutions for tomorrow?

We address the topic by inviting experts to discuss it in private and public forums - we create the environment for the people to meet and talk about this and by connecting them they will hopefully meet and start building solutions together or influence the decision makers in taking action.

Question: Can the ethical debate as well as data privacy, data protection and data diversity be framed more constructively? If yes, please share examples.

I am not sure there is a 'one size fits all' for this type of debate and people have different ways of approaching and understanding how data is changing their lives. I know a lot of people for example who are not interested in their own data privacy and protection, they prefer to donate their data for research and have more personalised products. In Europe data privacy and regulation have been important topic leading up to GDPR and now the formation of article 11 and 13 that will change drastically how information is shared on the web.

Question: How does Dataconomy help bridge the gap between data science and business? What are the two main challenges each side currently faces? How can both sides be brought closer together (eg legislators, 
corporations, startups, data managers, data subjects)?

We bridge the gap by conducting educational and training programs for all professionals to explain data science to non-techies and business managers as well as provide further training and workshops to data scientists. We also connect data scientists with career opportunities and assist businesses who do not currently have a data scientist in house or do not understand the power of their data to get consultancy and connect with the relevant stakeholders.

On one hand, data scientists who are currently recruited in business with a small or previously nonexistent data science department face the challenge of structuring, cleaning and optimising the existing data - this is a tedious and long procedure. Another challenge and this one should be addressed immediately and it is a challenge faced by both parties (data scientists and business professionals) is the lack of communication.

Business managers and professionals - you need to include data scientists in your vision and have them buy in. Data scientists as well as techies are not your regular 'working class' workforce. They should understand and embrace the vision of your company wholeheartedly. Also, the more involved you have them the more active and engaged they will become. This is certainly a way that both parties can come closer together.

A challenge uniquely faced by the business arm of a company is that the business managers don't speak the 'data science' lingo in which case I think a data dictionary should be established and all stakeholders should become data literate.

Question: Does the underrepresentation of women in data science influence how our society will look like tomorrow? Do you have examples that reveal the 'gender-tech-gap' especially in data science and in new data-based solutions businesses are building? How do you encourage women to work in technology?

The underrepresentation of any group and marginalised community establishes a system and an infrastructure where they are not properly represented and their input and experience is not properly factored in, so yes, it is important for any future systems that we are building to make them as inclusive and sustainable as possible. Women can be encour- aged to work in tech when and if the environments will take their experience into consideration; we need to include more female heroes in our lives, more female role models and narratives, more inclusive spaces, embracing and accepting the female and feminine traits of being and existing - currently all our systems are systems of patriarchy and we favour and encourage masculine and male characteristics and traits in the systems and infrastructures we built. We need to change our perception and mindset in order to encourage girls from a young age to code and become mathematicians, scientists, and programmers. We need to encourage women by fostering an environment that celebrates and invites them.

Question: What are the most interesting data-fused technologies/applications that currently inspire you? Why?

- Hippo.AI: A foundation started by Bart de Witte with the aim to democratise healthcare through supporting open source projects. It's something in between an NGO and an Open Source Foundation to advance humanity.

- Plan A: A data-driven platform in the fight against climate change

- KIProtect: The programme which enables data-driven teams to easily work with and share sensitive data with guaranteed security and compliance. Unlike most encryption methods, KIProtect detects and secures your data on the fly while retaining its usefulness and readability.

- The Data Union: The Data Union protects the interests of anyone who produces data on the internet. The Data Union wants to give people more control over their data and shape strong terms of conditions for organisation who use that data.

\section{Interview 2: Gapsquare - Revolutionise Fair Pay Through Al}

Name of your startup/initiative: Gapsquare Location: Bristol, UK

Your name, position: Dr Zara Nanu, CEO and Ant Kennedy, CTO

Question: What does Gapsquare do and what role does AI play? 
Gapsquare develops cloud based SaaS to help businesses avoid unfair pay structures. Our flagship product Gapsquare FairPay provides gender pay analysis, ethnicity pay, equal pay, and equal pay for equal value. Our vision has always been to use AI when we look at all the aggregated data to understand trends, explain gaps, and help companies close gaps that should not be there. The easier part is identifying (where they are present) any equal pay issues - and where employees are being paid differently, without any underlying reasons, for the same roles.

More complexities arise when we start looking at gender pay gaps, and identify reasons behind these gaps. Some issues that we are already beginning to identify are slow career progression for women, clustering of women in occupations that pay less per hour, and lack of flexible working.

This is where it gets more interesting as we bring together an interdisciplinary approach including social sciences, behavioral economics, and data science. It's a very exciting space. The World Economic Forum predicts it will take over 200 years to close the gender pay gap globally. We want to use data and tech to make this happen in 20 years.

This is where AI comes into play. By training computers to analyse large, complex data it can identify problematic indicators in seconds, and provide a foundation for scenario planning and fast identification of solutions. If done right, AI can help accelerate and make unprecedented progress.

Question: What are the benefits/potential of machines engineered to take decisions/process data? Feel free to include examples from your experience.

Initially we thought the machines are going to be the holy grail to solving pay inequalities at work. But it very quickly transpired that we are dealing with biased data, and that the algorithms themselves are also biased. If we apply these algorithms to existing data, all we will end up doing is accelerating inequality.

This is why we see cases such as the Amazon data science based recruitment platform. Amazon had a group of data scientists in Edinburgh develop a machine learning based tool to recruit talent into the company. The team created 500 computer models and was taught to recognise 50,000 terms that were found in their databases of past candidates and employees. As Amazon is a tech company, heavily reliant on a male workforce, the models began to favor male candidates and give lower scores to female candidates. The company had to scrap the program when they realised the bias.

What this shows is that companies are increasingly viewing machine learning as an obvious solution to business problems and equality problems in particular. But when not done right, this can significantly backfire.

Question: What are the challenges for ensuring that AI operaties safely, fairly and non-biased in the (near) future - what potential ethical considerations arise? How do Gapsquare or other organisations in your network deal with it?

Ethics is a very important part of our work. We operate with data that is embedded with bias. Data for hundreds of thousands of employees, with a large percentage of men in higher paid roles and women in lower paid roles, with more men in roles and departments that pay more per hour.

These problems don't only affect Gapsquare but all businesses that are using AI to achieve their goals. Although this may be a scary prospect, we at Gapsquare are tackling this through constant review of our AI systems and underlying datasets to alert where there might potentially be bias allowing us to take corrective action before exposing the technologies to our end users.

Question: What are your machine learning algorithms based upon? What type of decision-making process does it replicate? How do you integrate the data into your decision-making process of gapsquare?

Gapsquare FairPay allows us to augment existing human processes through continuous monitoring of salary data. This enables businesses to understand how they are tackling any issues they have in their pay structure. Using a companies internal data and anonymised and aggregated data from all of our customers, we can make recommendations on where you might have an unexplained pay gap, what business units, job roles contribute to you pay gap and recommend how you can approach closing these gaps.

Where our tool really becomes exciting is when we begin to add more data from organisations: such as performance reviews, exit interviews, etc. Gapsquare FairPay can then begin to make more intelligent decisions around individuals career progression 
with the tool working in collaboration with existing HR staff.

We are not looking to replace the human component of pay and reward decision-making. We view this as a process of co-working with AI in order to free up time for existing staff to tackle higher value problems whilst working to ensure the right people are in the right roles and they are remunerated fairly for their work.

Question: Based on your experience, how do you promote gender equality through technology?

Tech is still very much a mans world, as only $25 \%$ of the workforce in this occupation is female. Data from the World Economic Forum indicates we are far from closing the gender gap in tech (as well as any other Science, Technology, Engineering and Math occupations). This is an important subject because tech is increasingly re-shaping the world we live in. Predominately male teams are more likely to replicate gender bias and stereotypes when they develop AI and machine learning algorithms.

Although we are seeing progress and more young women are considering tech jobs, the rates of leaving the profession are growing. This is related to the fact that it is difficult for women to feel like they fit in in all male environments.

It will take a collective effort between the tech sector, educational systems and governments to create sustainable change. Companies can see through their data at Gapsquare where the key challenges are. Sometimes it is an issue of recruitment, other times an issue of career progression. Their data merely mirrors the global trends. A 2016 report from McKinsey shows that women made up $37 \%$ of entry-level roles in tech, and only $25 \%$ advanced to senior management roles.

Companies are starting to use tech to create fairer recruitment processes. For example by using tools that identify gender biased language in job ads before they are advertised, or the use of fair online recruitment platforms that remove people's names from the application process. But this is only half of the job in using tech to bring more women into the occupation. Educational establishments need to be creative in what technologies they use to attract female talent. Governments in turn have a role to play in developing and funding initiatives that bring women into tech.
Question: What traits will increasingly become important in the development of AI? List the top three according to you.

Trust. In a recent lightning Twitter Poll, I asked if readers trust Artificial Intelligence. I know, it was a 'sweeping generalisation' kind of question, in a world where AI and trust mean different things to different people, so any statistical accuracy is out the window. However, it was enough to show that an overwhelming majority don't have trust in AI (nearly $70 \%)$.

When we set up Gapsquare just over three years ago, we set off on a mission to use machine learning and Artificial Intelligence to create more diversity and inclusion in the workplace. Our aim is to use AI to create fairness in work.

While we are developing AI to help create more inclusion and diversity, we find increasingly that people do not necessarily trust it. At the end of the day, to the end user, $\mathrm{AI}$ is this black box in which you define what success looks like, mix it with some data, and hope that it delivers insights that will help you reach that goal.

The only way to overcome this big trust issue is transparency. And in order for it to be successful, transparency will have to cut across multiple levels - from collection and use of data to defining success together and looking at outcomes with an understanding that they can be flawed.

At the end of the day, AI is here to stay and if we do not build trust with its users, it will be developed in silos and applied in AI bubbles. Partial attempts towards transparency will not suffice. Trust will come with full disclosure, and tech giants will have to lead or follow on this. Trust will be built when data holders learn to embed transparency in what data they use, what kind of algorithms they use, and what outcomes are being generated.

Question: What do we need to do to teach morality to machines? How can we design more ethically aligned machines that maximise fairness and reduce biases? From your point of view what is needed to promote this? An interesting question connected to this is 'do we want to train AI to be like us or to be more moral than us? We've seen AI turn racist, mysogenic, homophobic, when we let it learn from us. And if we do decide that we want to teach an AI to be a mirror to our moral code, whose moral code should we use?' 
Morality means different things to different people and cultures. And by culture we mean it in the broadest sense as in the geography, but also in the more local sense as in the company culture. These cultures can play a big role in the way data scientists understand their remit in creating AI. These are all open questions to which we are contributing every day though our thinking and our decision making.

This was also apparent in the Moral Machine Experiment. The platform that generates moral dilemmas and collects information on the decisions that people make between two destructive outcomes. There are no right or wrong answers, but the data could be used to inform decision making inbuilt in self driving cars. What the research team found out is that while there are generic/global preferences when it comes to prioritising certain pedestrian over others when faced with a faulty self driving car, preferences revealed by the Moral Machine are highly correlated to cultural and economic variations between countries.

This is why decisions around morality of machines need careful consideration and thinking. Collectively we have to agree on an ethical framework and set of principles created for data practitioners and researchers. The framework will have to be based on trust and transparency if we want people to buy into it.

\section{Conclusion}

Without doubt, there is a lot of potential in data science, machine learning and artificial intelligence. Both interviews reveal the complexity of creating ethical solutions with a long-term positive impact on society. However, the also show how the ethical debate can add richness to the solutions that are being developed. Three key insights based on both interviews are: 1) A machine's 'morality' mirrors its creators. Accordingly, bias in data collection, data aggregation and decision-making processes makes it challenging to design fair and ethical solutions. Both startups emphasise the importance of critically evaluating every step of the process to reduce the risk of a solution turning bad.

2) Data science and artificial intelligence require a new form of awareness and collaboration among scientists, researchers, businesses as well as other stakeholders such as legislators, politicians and the public. Taking these different perspectives into account is essential to create solutions that will have a long-term positive impact on society.

3) More women have to be encouraged to get involved into this growing sector. Their ideas will create greater diversity and therewith empower the next generation to build less biased and more innovative solutions - ideally shaping a better future. 